[Article]

\title{
短链烷基对多取代烷基苯磺酸盐界面性质的影响
}

\author{
束宁凯 ${ }^{1}$ 徐志成 ${ }^{2}$ 刘子瑜 ${ }^{2}$ 靳志强 ${ }^{2}$ 黄建滨 $^{3}$ 张 磊 ${ }^{2}$ 张 路 ${ }^{2 *}$

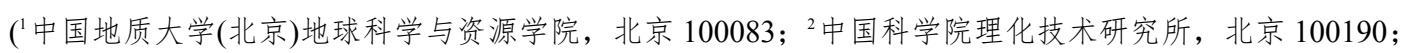 \\ ${ }^{3}$ 北京大学化学与分子工程学院, 北京 100871)
}

\begin{abstract}
摘要: 利用悬挂滴方法研究了 2,5-二乙基-4-壬基苯磺酸钠(292)、2,5-二丙基-4-壬基苯磺酸钠(393)和2,5-二丁 基-4-壬基苯磺酸钠(494)在空气-水表面和正癸烷-水界面的扩张流变性质, 考察了时间、界面压、工作频率及 体相浓度对扩张弹性和粘性的影响。研究发现, 在低表面活性剂浓度条件下, 表面吸附膜类似弹性膜, 其强 度由膜内分子的相互作用决定; 高浓度下体相与表面间的扩散交换过程控制表面膜的性质。油分子的插入导 致界面吸附分子之间相互作用的削弱, 扩散交换过程主导界面膜性质; 但随着短链烷基长度增加, 油分子的 影响变小。表面膜的强度在吸附达到平衡前已经决定, 而界面膜在吸附饱和后仍然随界面分子重排而变化。
\end{abstract}

关键词: 烷基苯磺酸盐; 表面; 界面; 扩张弹性; 扩张粘性

中图分类号: 0647

\section{Effect of Short Alkyl Chain on Interfacial Properties of Poly-Substituted Alkyl Benzene Sulfonate}

\author{
SHU Ning-Kai ${ }^{1} \quad$ XU Zhi-Cheng ${ }^{2} \quad$ LIU Zi-Yu ${ }^{2} \quad$ JIN Zhi-Qiang ${ }^{2}$ \\ HUANG Jian-Bin ${ }^{3} \quad$ ZHANG Lei ${ }^{2} \quad$ ZHANG Lu ${ }^{2, *}$ \\ ('School of Earth Sciences and Resources, China University of Geosciences (Beijing), Beijing 100083, P. R. China; \\ ${ }^{2}$ Technical Institute of Physics and Chemistry, Chinese Academy of Sciences, Beijing 100190, P. R. China; \\ ${ }^{3}$ College of Chemistry and Molecular Engineering, Peking University, Beijing 100871, P. R. China)
}

\begin{abstract}
The interfacial dilational rheological properties of 2,5-diethyl-4-nonyl benzene sulfonate(292), 2,5dipropyl-4-nonyl benzene sulfonate(393), and 2,5-dibutyl-4-nonyl benzene sulfonate(494) at air-water and decane-water interfaces were investigated by drop shape analysis. The influences of ageing time, interfacial pressure, dilational frequency, and bulk concentration on their dilational elasticity and viscosity were expounded. The surface adsorbed film was found to be elastic in nature at lower bulk concentration, and its strength determined by the interactions between the molecules in the film. In contrast, a diffusion-exchange process between the surface and the bulk controlled the properties of film at higher concentration. The insertion of oil molecules weakened the interactions among the adsorbed molecules, causing the diffusion-exchange process to dominate the nature of the interfacial film. However, this effect of the oil molecules decreased with increasing short alkyl chain length. The strength of the surface film could be determined before the adsorption equilibrium, while the nature of the interfacial film varied after saturated adsorption because of the re-arrangement of the interfacial surfactant molecules.
\end{abstract}

Key Words: Alkyl benzene sulfonate; Surface; Interface; Dilational elasticity; Dilational viscosity

Received: November 21, 2016; Revised: December 23, 2016; Published online: December 23, 2016.

*Corresponding author. Email: luyiqiao@hotmail.com; Tel: +86-10-82543589.

The project was supported by the National Science \& Technology Major Project, China (2011ZX05028).

国家科技重大专项(2011ZX05028)资助项目

(C) Editorial office of Acta Physico-Chimica Sinica 


\section{1 引言}

界面流变学主要涉及到应力、形变和形变速 率的关系, 它是评价界面膜的重要手段。根据形 变方式的不同, 界面流变学主要可分为两种：界 面剪切流变和界面扩张流变。界面剪切流变是通 过施加使界面形状发生改变而面积不发生变化的 外力, 考察剪切应力的响应, 从而获得界面层结 构和膜的机械强度的信息, 对吸附层内二维结构 的形成较为敏感 ${ }^{1-3}$; 而界面扩张流变是通过不改 变界面形状条件下对面积的规律扰动, 获得界面 动力学性质的相关参数, 从而了解界面上分子排 布、分子间相互作用和超分子聚集体的信息, 对 表面活性物质的吸附和脱附更为敏感 ${ }^{4-9}$ 。

烷基苯磺酸盐具有优良的界面活性和去污能 力, 是应用最为广泛的工业表面活性剂和家用高 泡洗涤剂, 同时, 也是提高石油采收率的重要化 学试剂 ${ }^{10}$ 。烷基链在苯环上的取代位置和取代数目 对于烷基苯磺酸盐的界面性质和应用性能有着重 要影响, 但尚缺乏系统、深入的研究。文献报道 了烷基支链化和多取代的长链烷基对烷基苯磺酸 盐界面行为及泡沫性能的影响 ${ }^{11-15}$, 本文则利用界 面扩张流变的实验手段, 研究了磺酸基邻位和间 位短链烷基对烷基苯磺酸盐在空气-水和正癸烷-水 界面上的吸附行为, 对揭示烷基苯磺酸盐类表面 活性剂的结构-性能关系具有重要意义。

\section{2 理论基础}

表面活性剂在表面上的吸附会降低溶液的表 面张力, 当吸附达平衡时, 表面张力降低至一平 台值。此时, 对表面吸附膜进行周期性(如正弦)的 面积扰动, 则表面张力也随之发生周期性(正弦)变 化。表面张力变化与表面面积相对变化的比值即 定义为表面扩张模量, 即

$$
\varepsilon=\frac{\mathrm{d} \gamma}{\mathrm{d} \ln A}
$$

其中, $\gamma$ 为表面张力, $A$ 为表面面积, $\varepsilon$ 为表面扩张
模量。

由于表面活性剂吸附膜中分子与体相间存在 扩散交换, 因此, 表面膜具有粘弹性。此时, 表 面张力的周期性变化与表面面积的周期性变化之 间存在相位差 $\theta$, 该相位差定义为表面扩张模量的 相角。表面扩张模量可用复数形式表示为:

$$
\varepsilon=\varepsilon_{\mathrm{d}}+\mathrm{i} \omega \eta_{\mathrm{d}}
$$

其中 $\omega$ 是表面面积正弦变化的频率。 $\varepsilon_{\mathrm{d}}$ (实数部分) 和 $\omega \eta_{\mathrm{d}}$ (虚数部分) 分别称为储存模量和损耗模量, 分别反映表面的弹性部分和粘性部分对粘弹性表 面膜性质的贡献。

表面扩张弹性和表面扩张粘度分别可按下式 计算:

$$
\begin{aligned}
& \varepsilon_{\mathrm{d}}=|\varepsilon| \cos \theta \\
& \eta_{\mathrm{d}}=\frac{|\varepsilon|}{\omega} \sin \theta
\end{aligned}
$$

\section{3 实验部分}

\section{1 实验材料}

表面活性剂 2,5-二乙基-4-壬基苯磺酸钠 (292)、2,5-二丙基-4-壬基苯磺酸钠(393)和 2,5-二丁 基-4-壬基苯磺酸钠(494), 均为实验室自制。分子 结构由 FT-IR 和 ${ }^{1} \mathrm{H}-\mathrm{NMR}$ 确定, 用两相滴定法测定 其纯度大于 $99.0 \%$, 具体结构见表 1 。合成细节见 文献 ${ }^{16}$ 。实验所用水为去离子水, 电阻率 $\geq 18$ $\mathrm{M} \Omega \cdot \mathrm{cm}$ 。正癸烷, 天津博迪化学有限公司, 分析纯。

\section{2 实验方法}

采用TRACKER 扩张流变仪(法国 IT-CONCEPT 公司生产)对悬挂气泡/液滴进行周期性振荡, 同时 利用滴外形分析方法实时测定气-液和液-液界面的 动态张力和扩张流变性质, 详细实验过程见文 献 ${ }^{11}$ 。实验温度为 $(30.0 \pm 0.1){ }^{\circ} \mathrm{C}$, 扩张形变 $(\triangle A / A)$ 为 $10 \%$, 水相为不同浓度的表面活性剂溶液。

\section{4 结果与讨论}

\section{1 表面吸附参数}

表 1 烷基苯磺酸钠的结构、名称及其缩写

Table 1 Structure, name and abbreviation of alkyl benzene sulfonates

\begin{tabular}{llllll}
\hline & No. & $m$ & $n$ & Sodium di-n-alkylbenzene sulfonate \\
\hline
\end{tabular}


测定了不同浓度 292、393 和 494 溶液的表面 张力, 通过表面张力对溶液摩尔浓度的对数作 图, 获得了 3 种表面活性剂的临界胶束浓度 $(\mathrm{cmc})$ 、临界胶束浓度时的表面张力 $\left(\gamma_{\mathrm{cmc}}\right)$ 以及极限 分子面积 $\left(A_{\min }\right)$, 实验结果见表 2 。从表中可以看 出, 随着疏水链的增长, 疏水作用增强, $\mathrm{cmc}$ 逐渐 降低, 表面活性增大。极限分子面积随疏水链长 的变化则正好相反, 这是因为分子结构中具有多 条疏水链, 因此, 极限分子面积主要取决于疏水 基的大小，随着疏水基总碳原子数的增加，疏水 基尺寸增大，这导致 494 的 $A_{\text {min }}$ 最大 ${ }^{17}$ 。不过，随着 磺酸基邻位和间位短链烷基的增长, 表面甲基密 度增大, 因此尽管吸附量有所降低, 但表面张力 仍然明显降低。

\section{2 动态表面张力及动态表面扩张流变性质}

动态表面张力反映了多取代烷基苯磺酸盐在 表面上的动态吸附过程, 而动态流变参数则反映 了吸附膜形成过程中的相互作用和膜强度的变 化。本文研究的 3 种烷基苯磺酸盐结构类似，其动 态表面张力及表面扩张参数的变化趋势较相似, 因此, 文中用 494 溶液的动态参数作为代表。不同 浓度 494 溶液动态表面张力和动态表面扩张弹性见 图1。从图1(A)中可以看出, 低浓度时, 在很长一 段时间内表面张力维持一个接近溶剂表面张力的 较高值, 然后表面张力下降, 整个曲线呈现 “ $\mathrm{S}$ ” 型。随着体相浓度增大，扩散交换加快并控制了表 面张力的变化, 因此, 达到平衡的时间明显缩短。

比较图 1(A)和图 1(B), 可以发现表面张力和 扩张弹性随时间变化的转折点比较一致。实验初 期, 表面吸附的分子较少, 表面张力接近于纯水 的表面张力值, 扩张模量接近于零; 随着浓度的 增大，表面上吸附的分子明显增多，表面张力快 速下降, 扩张弹性随之显著增大; 当吸附接近平 衡时, 表面张力变化缓慢, 而扩张弹性也达到相 应的平台值。这说明多取代烷基苯磺酸盐的表面 吸附膜的性质与吸附量正相关，不存在 “亚层”

表 $230{ }^{\circ} \mathrm{C}$ 时表面活性剂的临界胶束浓度、极限分子面积 和临界胶束浓度时的表面张力

Table 2 cmc, limit of molecular area $\left(A_{\min }\right)$ and surface tension $\left(\gamma_{\mathrm{cmc}}\right)$ of the studied surfactants at $30^{\circ} \mathrm{C}$

\begin{tabular}{cccc}
\hline No. & $10^{4} \mathrm{cmc} /\left(\mathrm{mol} \cdot \mathrm{L}^{-1}\right)$ & $A_{\min } / \mathrm{nm}^{2}$ & $\gamma_{\mathrm{cm} /}\left(\mathrm{mN} \cdot \mathrm{m}^{-1}\right)$ \\
\hline 292 & 9.49 & 1.07 & 35.6 \\
393 & 3.38 & 1.17 & 31.7 \\
494 & 0.63 & 1.40 & 30.2 \\
\hline
\end{tabular}
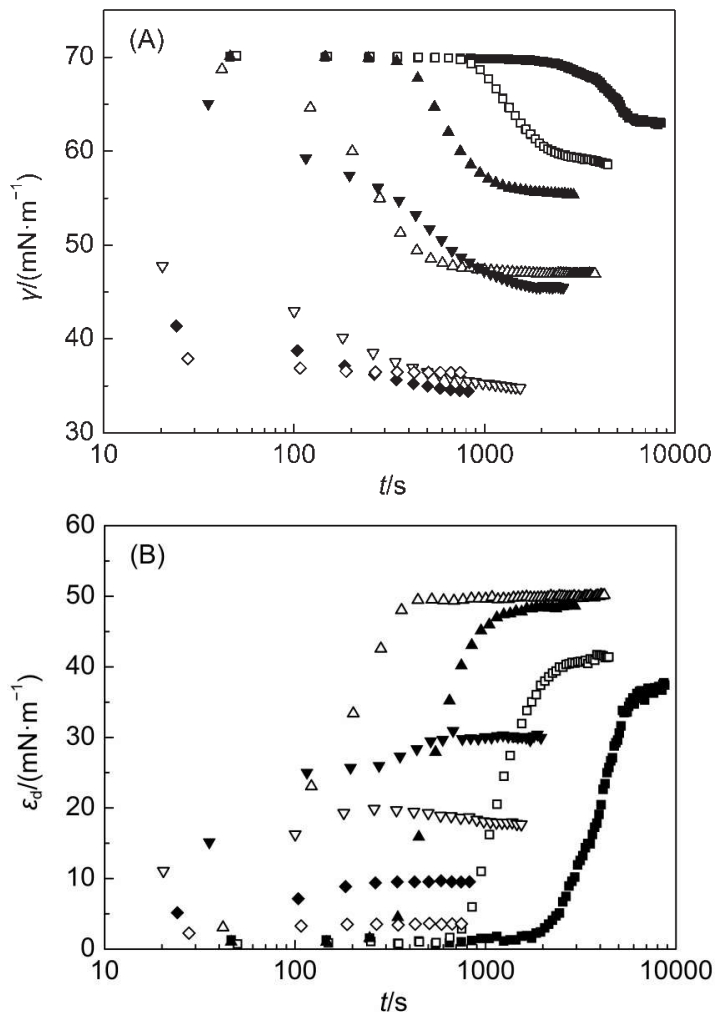

图 1 不同浓度 494 溶液的动态表面张力(A)、 动态表面扩张弹性(B)

Fig.1 Dynamic surface tension (A) and surface dilational elasticity (B) of 494 solutions with various concentrations

$c_{(494)} /\left(\mathrm{mol} \cdot \mathrm{L}^{-1}\right): \square 1 \times 10^{-7} ; \square 5 \times 10^{-7} ; \mathbf{\Delta} 1 \times 10^{-6} ; \triangle 5 \times 10^{-6} ;$

$\nabla 1 \times 10^{-5} ; \nabla 6.3 \times 10^{-5} ; \diamond 1.3 \times 10^{-4} ; \diamond 3.2 \times 10^{-4}$

结构。

为深入了解表面吸附膜的特征行为, 可以将 表面扩张弹性和粘性对表面压作图, 如图 2所示。

扩张参数-表面压曲线是探索吸附膜特性的重 要手段。文献中报道，不同浓度蛋白质的扩张参 数-表面压曲线几乎重合在一起，并经过一个极大 值，该曲线反映了蛋白质吸附膜随分子表面浓度 变化而经历的分子单独吸附、折叠分子展开、形 成表面有序结构、出现表面亚层等结构变化过 程 ${ }^{18}$ 。这是因为对于蛋白质分子，表面与体相间的 扩散交换几乎可以忽略，表面压直接取决于吸附分 子的数目和分子的排列方式，与体相浓度无关。而 对于一般的小分子表面活性剂, 扩散交换过程决定 了表面膜的性质，在同一表面压时，扩张参数随 体相浓度的增大而降低，因此不同浓度溶液的表 面压与扩张参数的曲线是呈分散状态的 ${ }^{19}$ 。

从图 2 可以看出，当表面活性剂浓度低于 $5 \times$ $10^{-6} \mathrm{~mol} \cdot \mathrm{L}^{-1}$ 时，不同浓度溶液的扩张弹性和粘性 对表面压的曲线重叠在一起，说明此时扩散交换 

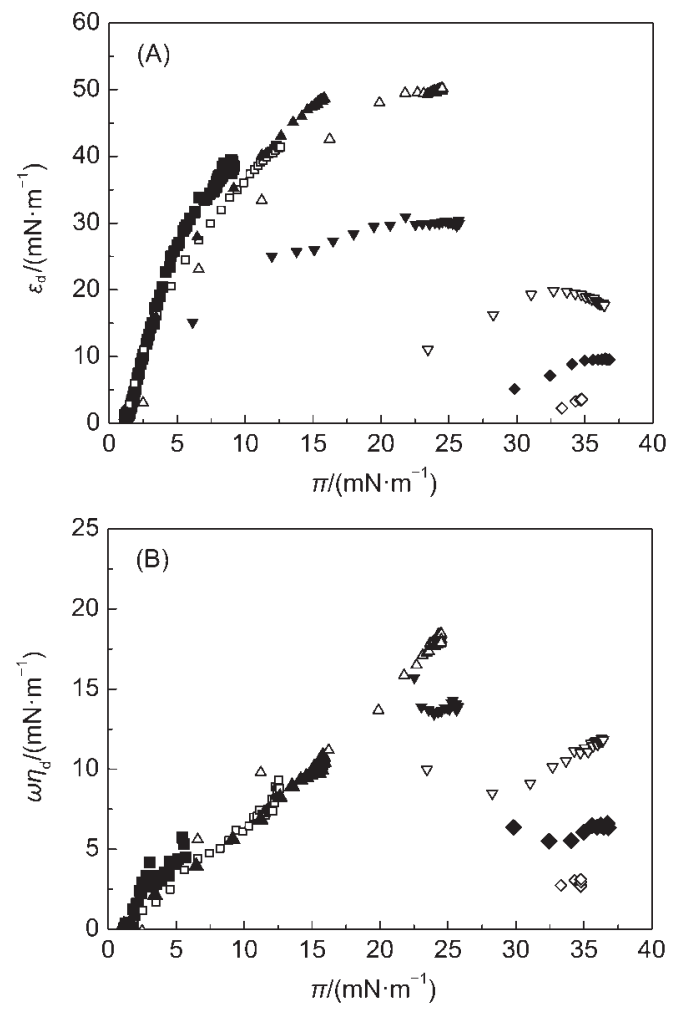

图 2 不同浓度 494 溶液的表面扩张弹性-表面压(A)和 粘性-表面压(B)曲线

Fig.2 Surface dilational elasticity (A) and viscosity (B) as a function of surface pressure for 494 solutions with various concentrations

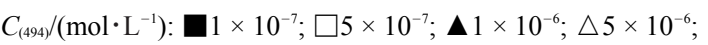

$\nabla 1 \times 10^{-5} ; \nabla 6.3 \times 10^{-5} ; \diamond 1.3 \times 10^{-4} ; \diamond 3.2 \times 10^{-4}$

对表面膜影响较小, 其性质由膜内分子的行为决 定。浓度进一步升高, 体相与表面间的扩散交换 过程控制表面膜的性质, 曲线随浓度增大呈分散 状态, 且同一表面压时, 体相浓度越大, 扩张弹 性和粘性越小。

另外, 对于实验范围内的所有浓度的溶液, 当吸附接近平衡时, 弹性和粘性的变化幅度远小 于表面压的变化幅度。这说明表面吸附膜的粘弹 特性在吸附达到平衡前就已决定，而表面张力的 降低则直到吸附量不再变化时才保持不变 ${ }^{20}$ 。这是 由于当具有一定强度的吸附膜形成后, 继续增加 表面活性剂分子的表面浓度, 虽然能够增加表面分 子间的相互作用，但同样增大扩散交换过程对表面 膜的影响, 因此并不能对表面膜的强度有贡献。

\section{3 工作频率对表面扩张性质的影响}

扩张实验的工作频率是影响扩张流变参数的 重要因素, 文中考察了频率范围 0.005-0.1 Hz 对弹 性和粘性的影响, 实验结果如图3 所示。
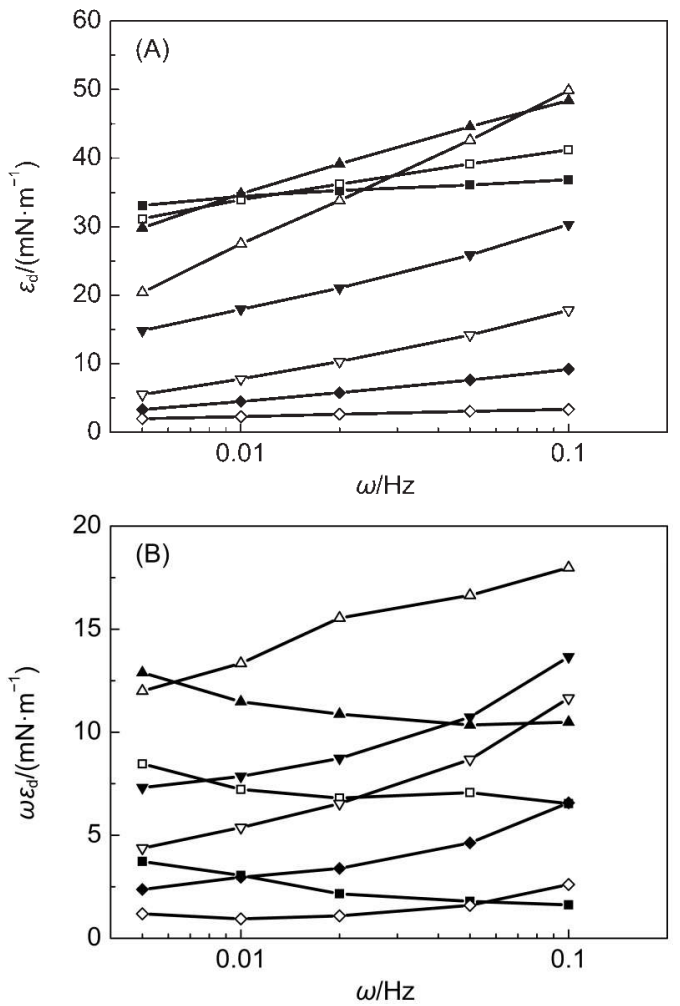

图 3 不同浓度 494 溶液的表面扩张弹性(A)和粘性(B) 随工作频率的变化

Fig.3 Surface dilational elasticity (A) and viscosity (B) as a function of frequency for $\mathbf{4 9 4}$ solutions with various concentrations

$C_{(494)} /\left(\mathrm{mol} \cdot \mathrm{L}^{-1}\right): \square_{1} \times 10^{-7} ; \square 5 \times 10^{-7} ; \mathbf{\Delta} 1 \times 10^{-6} ; \triangle 5 \times 10^{-6} ;$

$\nabla 1 \times 10^{-5} ; \nabla 6.3 \times 10^{-5} ; \diamond 1.3 \times 10^{-4} ; \diamond 3.2 \times 10^{-4}$

扩张弹性直接与表面分子间的相互作用相 关, 相互作用越强, 弹性越大。同时, 表面及其 附近的所有弛豫过程损耗能量, 降低表面弹性。 因此, 频率越高, 弛豫过程的作用越弱, 弹性就 越强。只有对不存在弛豫过程的纯弹性膜, 或者 弛豫过程特征频率远低于工作频率的吸附膜, 其 弹性才不随频率改变而变化 ${ }^{21}$ 。从图3(A)中可以看 出, 当表面活性剂浓度低于 $5 \times 10^{-6} \mathrm{~mol} \cdot \mathrm{L}^{-1}$ 时, 表 面弹性和浓度的双对数曲线近乎平行, 表面膜类 似弹性膜。这与前面表面弹性-表面压曲线显示的 结果是一致的。而随着浓度增大, 扩张弹性的频 率依赖性增强, 显示为递增的趋势, 说明此时表 面膜呈粘弹性，扩散交换过程决定表面膜的性质。

一般认为, 扩张粘性反映界面上及界面附近 各种号豫过程的总和, 并且不同的弛豫过程都会 有各自的特征频率, 粘性在特征频率处出现最大 值。494 溶液表面扩张粘性随频率的变化见图 3 (B)。从图中可以看出, 当表面活性剂浓度低于 $5 \times$ 
$10^{-6} \mathrm{~mol} \cdot \mathrm{L}^{-1}$ 时, 扩张粘性随频率而降低, 而高浓 度时的变化趋势正好相反。这说明低浓度时表面 膜由特征频率低于 $0.005 \mathrm{~Hz}$ 的慢过程决定, 表面扩 张粘性出现最大值的频率要低于工作频率。而高 浓度时, 特征频率高于 $0.1 \mathrm{~Hz}$ 的扩散交换弛豫过程 控制表面膜的性质 ${ }^{22}$ 。

\section{4 表面活性剂浓度对表面扩张性质的影响}

浓度是影响扩张性质的另外一个重要因素。 工作频率为 $0.1 \mathrm{~Hz}$ 时，292、393、494 溶液的表面 扩张弹性和粘性随浓度的变化趋势见图 4。从图中 可以看出, 3 种多取代烷基苯磺酸盐溶液的表面扩 张弹性和粘性均随浓度增大通过最大值, 出现最 大值的浓度均低于其 $\mathrm{cmc}$ ，这是体相浓度增加同时 增大界面吸附量和增强扩散交换过程的综合作用 的结果。有趣的是, 随着短链烷基的增长, 扩张 弹性的最大值仅略有升高, 而扩张粘性的最大值 则几乎不变。这是由于对于多取代烷基苯磺酸盐 而言，随着短链烷基的增长，表面吸附分子之间 的相互作用与烷基链之间的交叠和取向变化得到
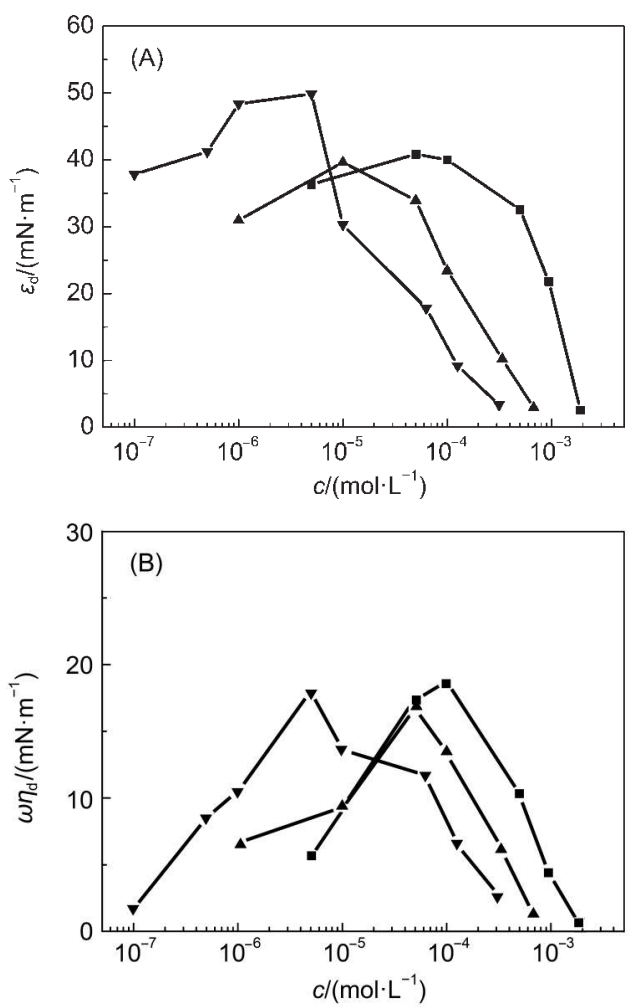

图 4292,393 和 494 溶液的表面扩张弹性(A)和 扩张粘性(B)随浓度的变化

Fig.4 Surface dilational elasticity (A) and viscosity (B) as a function of concentration for 292, 393 and 494 solution concentrations frequency: $0.1 \mathrm{~Hz} ; \mathbf{\square} 292 ; \boldsymbol{\Delta} 393 ; \boldsymbol{\nabla} 494$
增强; 但表面吸附量由于位阻效应而降低。这两 方面的变化对表面扩张参数的影响趋势相反, 彼 此抵消, 造成实验研究的不同链长烷基苯磺酸盐 的表面扩张参数变化不大。

\section{5 界面压与扩张弹性及扩张粘性的关系曲线}

为了更深刻地了解多取代烷基苯磺酸盐在流 体界面的行为, 考察了其在正癸烷-水界面上的扩 张流变性质。3 种烷基苯磺酸盐的动态界面张力和 动态界面扩张流变参数的变化规律与表面类似, 不再赘述。不同浓度 494 溶液的界面扩张弹性和粘 性的界面压曲线如图 5 所示。

从图中可以看出, 总体而言, 494 的界面弹性 和粘性的界面压曲线均呈分散状态, 意味着扩散 交换过程控制界面膜的性质, 这与表面膜截然不 同。这是由于油分子插入到界面上的烷基苯磺酸 盐分子之间, 在一定程度上削弱分子间相互作 用, 促进界面分子与体相分子之间的交换, 扩散 交换过程得到加强。另外, 弹性和粘性的界面压
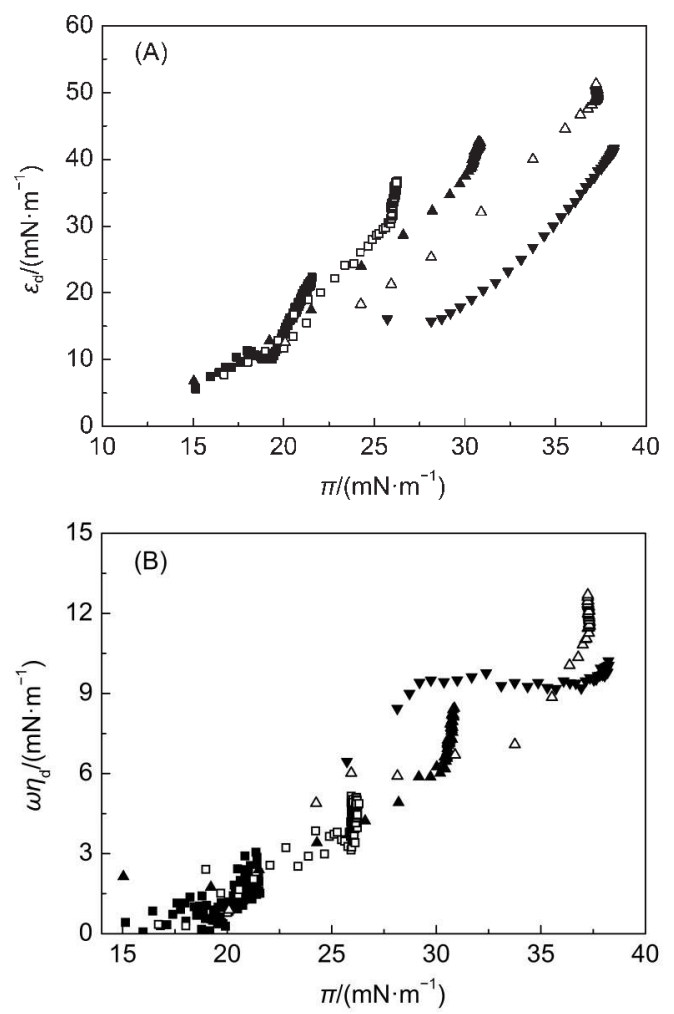

图 5 不同浓度 494 溶液的界面扩张弹性-界面压关系(A)和 界面扩张粘性-界面压关系(B)

Fig.5 Interfacial dilational elasticity (A) and viscosity (B) as a function of interfacial pressure for $\mathbf{4 9 4}$ solutions with various concentrations

oil phase: decane; $c_{(494)} /\left(\mathrm{mol} \cdot \mathrm{L}^{-1}\right): \boldsymbol{\square} 1 \times 10^{-7} ; \square 5 \times 10^{-7}$; $\Delta 1 \times 10^{-6} ; \triangle 5 \times 10^{-6} ; \mathbf{\nabla} 1 \times 10^{-5}$ 
曲线的尾端呈近乎垂直的状态, 说明多取代烷基 苯磺酸盐在吸附量达到饱和、界面张力几乎不变 的状况下, 仍然存在界面分子的重排, 界面膜的 强度继续增加。

\section{6 表面活性剂浓度对界面扩张性质的影响}

工作频率为 $0.1 \mathrm{~Hz}$ 时，292、393、494 溶液的 界面扩张弹性和粘性随浓度的变化趋势见图6。从 图中可以看出, 3 种多取代烷基苯磺酸盐溶液的界 面扩张弹性和粘性也随浓度增大通过最大值。与 表面不同的是, 随着短链烷基的增长, 扩张弹性 明显升高, 扩张粘性略有增大。这是由于界面膜 主要由扩散交换过程主导, 随着碳链增加, 分子 尺寸增大, 扩散交换变慢, 界面形变过程中能量 损耗降低, 因而扩张弹性升高。同时, 烷基链变 长, 其取向变化过程的贡献增大, 粘性有所增加。

对比图 4 和图 6 可以发现, 油分子插入到界面 上吸附的烷基苯磺酸盐分子之间, 破坏短链烷基 之间的相互作用, 造成扩张弹性的降低; 但随着 短链烷基的增长, 这种破坏作用逐渐变弱, 494 溶
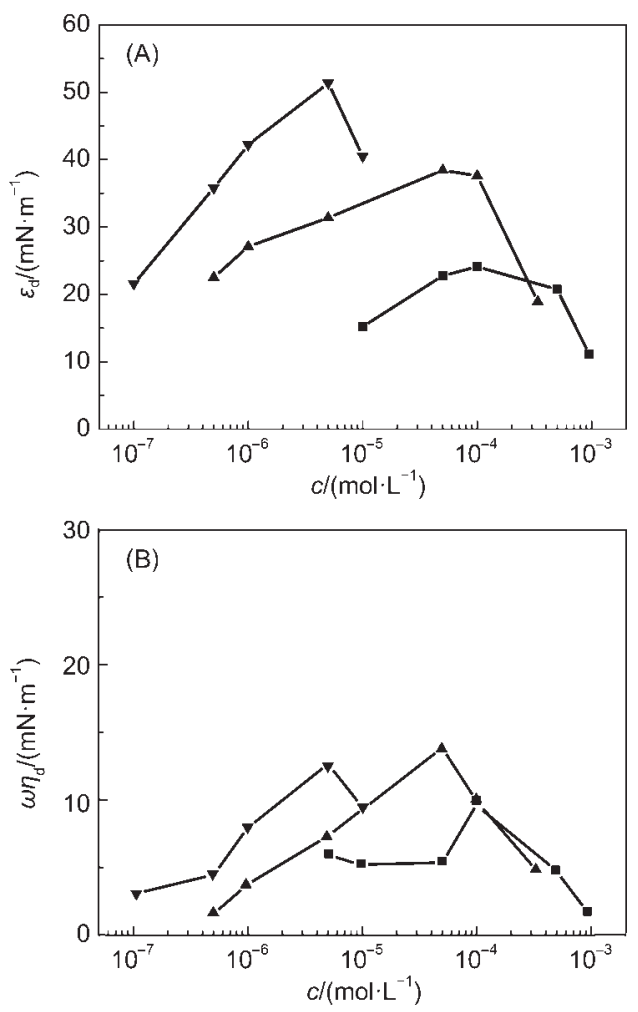

图 6292,393 和 494 溶液的界面扩张弹性(A)和 扩张粘性(B)随浓度的变化

Fig.6 Interfacial dilational elasticity (A) and viscosity (B) as a function of concentration for 292, 393 and 494 solutions

oil phase: decane; frequency: $0.1 \mathrm{~Hz} ; \mathbf{\square} 292 ; \boldsymbol{\Delta} 393 ; \mathbf{\nabla} 494$
液的表面弹性最大值和界面弹性最大值几乎相 等。说明一定长度的短链烷基能让油分子的插入 变得困难。而 3 种烷基苯磺酸盐的界面粘性均明显 低于表面粘性, 则显示油分子对界面内弛豫过程 的削弱更加明显。

\section{5 结 论}

考察了磺酸基邻位和间位的短链烷基对多取 代烷基苯磺酸盐在空气-水表面和正癸烷-水界面的 扩张流变性质的影响, 研究发现: 在低表面活性 剂浓度条件下, 多取代烷基苯磺酸盐的表面吸附 膜类似弹性膜, 其强度由膜内分子的相互作用决 定; 高浓度下, 体相与表面间的扩散交换过程控 制表面膜的性质。随着短链烷基长度增加, 表面 膜的弹性略有增加, 粘性变化不大。

油分子的插入导致界面吸附分子之间相互作 用的削弱, 扩散交换过程主导界面膜性质, 界面 膜的弹性随着长度增加而明显增大。短链烷基增 加至一定长度, 油分子插入变得困难, 表面膜和 界面膜的弹性接近相等。表面膜的强度在吸附达 到平衡前已经决定, 而界面膜的强度在吸附饱和 后仍然随吸附分子重排而明显增大。

\section{References}

(1) Miller, R.; Ferri, J. K.; Javadi A.; Kragel, J.; Mucic, N.; Wustneck, R. Colloid Polym. Sci. 2010, 288 (9), 937. doi: 10.1007/s00396-010-2227-5

(2) Kraegel, J.; Derkatch, S. R. Curr. Opin. Colloid Interface Sci. 2010, 15 (4), 246. doi: 10.1016/j.cocis.2010.02.001

(3) Patino, J. M. R.; Fernández, M. C.; Sánchez, C. C.; NiÖO, M. R. R. J. Colloid Interface Sci. 2007, 313 (1), 141. doi: 10.1016/j. jcis.2007.04.025

(4) Murray, B. S. Curr. Opin. Colloid Interface Sci. 2002, 7 (5-6), 426. doi: 10.1016/S1359-0294(02)00077-8

(5) Miller, R.; Fainerman, V. B.; Makievski, A. V.; Krägel, J.; Grigoriev, D. O.; Kazakov, V. N.; Sinyachenko; O. V. $A d v$. Colloid Interface Sci. 2000, 86 (1-2), 39. doi: 10.1016/S00018686(00)00032-4

(6) Noskov, B. A.; Akentiev, A. V.; Bilibin, A. Y.; Zorin, I. M.; Miller, R. Adv. Colloid Interface Sci. 2003, 104, 245. doi: 10.1016/S0001-8686(03)00045-9

(7) Ivanov, I. B.; Danov, K. D.; Ananthapadmanabhan, K. P.; Lips, A. Adv. Colloid Interface Sci. 2005, 114-115, 61. doi: 10.1016/j. cis.2004.11.001

(8) Wang, Y. Y.; Zhang, L.; Sun, T. L.; Fang, H. B.; Zhao, S.; Yu, J. Y. Acta Phys. -Chim. Sin. 2003, 19 (4), 297. [王宜阳, 张 路, 孙 涛垒, 方洪波, 赵 濉, 俞稼镛. 物理化学学报, 2003, 19 (4), 
297.] doi: 10.3866/PKU.WHXB20030403

(9) Cao, X. L.; Feng, J.; Guo, L. L.; Zhu, Y. W.; Zhang, L.; Zhang, L.; Luo, L.; Zhao, S. Colloids Surf. A 2016, 490, 41. doi: 10.1016/j.colsurfa.2015.11.030

(10) Zhao, G. X.; Zhu, B.Y. Principles of Surfactant Action; Beijing: China Light Industry Press, 2003. [赵国罕, 朱㻉瑶. 表面活性 剂作用原理. 北京: 中国轻工业出版社, 2003.]

(11) Zhang, L.; Wang, X. C.; Gong, Q. T.; Luo, L.; Zhang, L.; Zhao, S.; Yu, J. Y. Acta Phys. -Chim. Sin. 2007, 23 (10), 1652. [张 磊, 王晓春, 宫清涛, 罗 澜, 张 路, 赵 濉, 俞稼镛. 物理化 学学报, 2007, 23 (10), 1652.] doi: 10.3866/PKU. WHXB20071031

(12) Zhang, L.; Wang, X. C.; Gong, Q. T.; Luo, L.; Zhang, L.; Zhao, S.; Yu, J. Y. J. Colloid Interface Sci. 2008, 327 (2), 451. doi: 10.1016/j.jcis.2008.08.019

(13) Ma, B. D.; Zhang, L.; Gao, B. Y.; Zhang, L.; Zhao, S.; Yu, J. Y. Colloid Polym. Sci. 2011, 289 (8), 911. doi: 10.1007/s00396011-2415-y

(14) Zhang, J. C.; Guo, L. L.; Zhang, L.; Zhang, L.; Luo, L.; Zhao, S. Z. Phys. Chem. 2013, 227 (4), 429. doi: 10.1524/ zpch.2013.0373

(15) Song, X. W.; Zhang, L.; Cao, X. L.; Li, Z. Q.; Zhang, L.; Luo, L.; Zhao, S. Colloids Surf. A 2012, 412, 64. doi: 10.1016/j. colsurfa.2012.07.016
(16) Gong, Q.T. Study on the Synthesis of Sodium Multi- $n$ Alkylbenzene Sulfonates and Their Properties of Interface and Solutions. Ph. D. Dissertation. Beijing: Institute of Physics and Chemistry, Chinese Academy of Sciences, 2005. [宫清涛. 多取 代直链烷基苯磺酸钠的合成及其界面与体相性质的研究. [D]. 北京: 中国科学院理化技术研究所, 2005.]

(17) Cui, X. H.; Wang, L.; Cao, X. L.; Zhao, F. L.; Luo, L.; Zhang, L.; Zhao, S.; Yu, J. Y. J. Dispersion Sci. Technol. 2008, 29 (8), 1153. doi: $10.1080 / 01932690701817990$

(18) Bos, M. A.; van Vliet, T. Adv. Colloid Interface Sci. 2001, 91 (3), 437.

(19) Huang, Y. P.; Zhang, L.; Zhang, L.; Luo, L.; Zhao, S.; Yu, J. Y. J. Phys. Chem. B 2007, 111 (20), 5640. doi: 10.1021/jp070997t

(20) Li, X. L.; Zhang, L.; Gong, Q. T.; Zhang, L.; Zhao, S.; Yu, J. Y. Acta Phys. -Chim. Sin. 2010, 26 (3), 631. [李秀兰, 张 否, 宫清 涛, 张 路, 赵 濉. 俞稼镛. 物理化学学报, 2010, 26 (3), 631.] doi: 10.3866/PKU.WHXB20100319

(21) Sun, H. Q.; Zhang, L.; Li, Z. Q.; Zhang, L.; Luo, L.; Zhao, S. Soft Matter 2011, 7 (17), 7601. doi: 10.1039/c1sm05234a

(22) Zhang, L.; Wang, X. C.; Gong, Q. T.; Luo, L.; Zhang, L.; Zhao, S.; Yu, J. Y. Acta Phys. -Chim. Sin. 2007, 23 (12), 1881. [张 磊, 王晓春, 宫清涛, 罗 澜, 张 路, 赵 濉, 俞稼镛. 物理化 学学报, 2007, 23 (12), 1881.] doi: 10.3866/PKU. WHXB20071210 